\title{
Survey of Incidence and Nested PCR Detection of Sugarcane White Leaf in Different Varieties
}

\author{
Rong-Yue Zhang, Hong-Li Shan, Ying-Kun Huang, ${ }^{\dagger}$ Xiao-Yan Wang, Jie Li, Wen-Feng Li, Xiao-Yan Cang, Jiong Yin, \\ and Zhi-Ming Luo
}

Sugarcane Research Institute, Yunnan Academy of Agricultural Sciences, Yunnan Key Laboratory of Sugarcane Genetic Improvement, Kaiyuan 661699, P.R. China

\begin{abstract}
Sugarcane white leaf (SCWL) is a devastating sugarcane (Saccharum officinarum) disease caused by a $16 \mathrm{SrXI}$ group phytoplasma, which is extremely harmful to sugarcane production. To determine the occurrence of SCWL in different varieties in 2018, we conducted a field survey and performed nested PCR detection of SCWL phytoplasma in cane-planting areas of Mangweng and Hepai in Gengma, Yunnan province, which are the areas most severely affected by SCWL in China. The results of the field survey showed that the symptomatic in-

SCWL phytoplasma was detected in symptomatic plants of all varieties more than $90 \%$ of the time; the SCWL phytoplasma was detected in 91 and $97 \%$ of symptomatic plants of Yingyu91-59 and Liucheng05-136 varieties, respectively. The SCWL phytoplasma was detected by PCR in $82 \%$ of the asymptomatic plant samples. The results of this study showed that field survey based on white leaf symptoms did not accurately reflect the actual occurrence of the SCWL phytoplasma.
\end{abstract} cidence of SCWL differed among varieties. The mean symptomatic incidence of SCWL on variety Yuetang60 was the highest (73.50\%), and it was the lowest on Liucheng05-136 (13.67\%). Using nested PCR, the
Keywords: phytoplasma, sugarcane white leaf, disease symptoms, incidence rate, resistant varieties
Sugarcane white leaf (SCWL) is a devastating disease of sugarcane (Saccharum officinarum) caused by a $16 \mathrm{SrXI}$ group phytoplasma (Zhang et al. 2016). The main symptoms of SCWL are soft leaves, a decreased chlorophyll content and leaf whitening, increased tillering, dwarf plants, shortened internodes, and clustered top leaves. After sugarcane is infected with SCWL phytoplasma, plant height, stem diameter, effective stem, and single-stem weight decrease significantly, resulting in a serious loss of sugarcane yield and sugar content (Huang et al. 2018). The SCWL phytoplasma is mainly spread by the transport of diseased cane planting materials and can also be transmitted by leafhoppers in the field (Hanboonsong et al. 2002, 2006; Rao et al. 2017; Tiwari et al. 2017). SCWL was first reported in Thailand in 1954 (Marcone 2002) and has been widely distributed in sugarcane producing countries such as India, Pakistan, Sri Lanka, the Philippines, Myanmar, and Vietnam, and it causes huge economic losses to the local sugarcane and sugar industries (Kumarasinghe and Jones 2001; Thein et al. 2012; Wang et al. 2015; Wongkaew et al. 1997). In China, presence of SCWL was recently confirmed by nested PCR in Baoshan, Yunnan province ( $\mathrm{Li}$ et al. 2013). Subsequently, SCWL was identified in Lincang and Puer, Yunnan province, and has spread rapidly (Zhang et al. 2016, 2019), seriously damaging sugarcane yield and sugar content and posing a major threat to sugarcane production.

Although SCWL is currently only found in some sugarcane planting areas in Yunnan province, the area affected by SCWL is gradually

\section{${ }^{\dagger}$ Corresponding author: Y.-K. Huang; huangyk64@163.com}

Funding: This work was supported by the National Natural Science Foundation of China (31760504), the Sugar Crop Research System (CARS-170303), Yunling Industry and Technology Leading Talent Training Program "Prevention and Control of Sugarcane Pests" (2018LJRC56), Yunnan Agricultural Foundation Projects (2017FG001[-054]), and the Yunnan Province Agriculture Research System.

The author(s) declare no conflict of interest.

Accepted for publication 6 April 2020.

(C) 2020 The American Phytopathological Society expanding. The results of previous field observations based on visual assessment of disease symptoms indicate that the area affected by SCWL in Shidian county and Longyang district in Baoshan, Yunnan province, expanded to 80 ha in 2012. In 2013, the areas of SCWL occurrence amounted to over 300 ha in Gengma county, Zhenkang county, Shuangjiang county, and Linxiang district in Yunnan province (Li et al. 2014a). Previous surveys to estimate the SCWL incidence of affected major sugarcane varieties in the main diseased areas of Baoshan and Lincang in Yunnan province documented that disease incidence and the degree of damage to different sugarcane varieties differed. Disease incidence of SCWL-resistant varieties was less than $10 \%$, whereas SCWL-susceptible varieties had disease incidences ranging from 50 to 100\% (Li et al. 2013, 2014b). Although preliminary observations have been made concerning the incidence of disease symptoms on different sugarcane varieties, the incidence of the SCWL phytoplasma in different varieties has not been systematically studied, and grading standards and evaluation methods of sugarcane resistance to the SCWL phytoplasma have not been established. In 2018, we surveyed the occurrence of SCWL and collected 780 samples in Mangweng and Hepai in Gengma, which are the areas most seriously affected by SCWL. The aim of this study was to determine if sugarcane varieties vary in symptom expression associated with SCWL phytoplasma infection by comparing the incidence of SCWL disease symptoms to the incidence of SCWL phytoplasma detection in different sugarcane varieties. Our goal was to lay a foundation for establishing resistance grading standards and evaluation methods for the management of SCWL phytoplasma.

\section{Materials and Methods}

SCWL survey and sample collection. In May 2018, a survey of SCWL occurrence and sample collection was carried out in Mangweng and Hepai in Gengma, which are the areas most severely affected by SCWL in Yunnan province. Samples were collected from the representative main varieties. In total, 26 plots were surveyed. Three rows were randomly selected within each plot, and the incidence of SCWL among 100 sugarcanes was continuously surveyed for the presence or absence of SCWL symptoms at each sampled row. A total of 300 plants were surveyed for all three rows, the number of plants presenting SCWL symptoms was recorded, and the SCWL incidence rate was calculated. The last 10 plants of 100 plants surveyed were continuously sampled, and each sample 
was numbered and its symptoms recorded. The first fully expanded young leaf from each sample was collected.

DNA extraction and nested PCR. DNA was extracted from $0.2 \mathrm{~g}$ of leaf material per sample using the Easy Pure Plant Genomic DNA Kit (TransGen Biotech, Shanghai, China) according to the manufacturer's instructions. Nested PCR assays were carried out using phytoplasma universal primer pair P1/P7 (Smart et al. 1996) followed by R16F2n/R16R2 (Lee et al. 1998). P1/P7 was used as the first set of primers in the first PCR reaction. The thermal cycling conditions were as follows: $3 \mathrm{~min}$ at $94^{\circ} \mathrm{C}$ followed by 35 cycles for $30 \mathrm{~s}$ at $94^{\circ} \mathrm{C}, 30 \mathrm{~s}$ at $55^{\circ} \mathrm{C}$, and $1 \mathrm{~min}$ at $72^{\circ} \mathrm{C}$, with a final extension for $10 \mathrm{~min}$ at $72^{\circ} \mathrm{C}$. For nested PCR, $1 \mu \mathrm{l}$ of the diluted first PCR product (1:30) was used as a template, and the annealing temperature was $57^{\circ} \mathrm{C}$. The PCR products were electrophoresed through a $1.5 \%$ agarose gel, stained in ethidium bromide, and visualized with a UV transilluminator. The final nested PCR product size was about $1,247 \mathrm{bp}$.

Cloning and sequencing of PCR products. One nested PCR product from the 10 samples from each surveyed plot was randomly selected for sequencing. Twenty-six PCR products were purified using a nucleic acid purification kit (Tiangen Biotech, Beijing, China) and cloned into the pEASY-T5 vector (Tiangen Biotech). The recombinant plasmids were introduced into Escherichia coli strain DH5a. Six positive clones were selected for each transformation, and the resulting DNA was sent to BGI Sequencing Co. (Beijing, China) for sequencing.

\section{Results}

Incidence of SCWL in the major sugarcane varieties. The incidence of SCWL symptoms in seven local major sugarcane varieties was analyzed in the field (Fig. 1). The variety that showed the highest SCWL incidence was Yuetang60, with an incidence of 73.50\% (441/ 600 ), and the lowest incidence of $13.67 \%$ (41/300) was found for Liucheng05-136. Nested PCR showed that the SCWL phytoplasma detection rate of all varieties was more than $90 \%$; the variety with lowest SCWL phytoplasma detection rate (90.95\% [191/210]) was Yingyu91-59, and the variety with the highest SCWL phytoplasma detection rate (96.67\% [29/30]) was Liucheng05-136.

SCWL phytoplasma detection rate of asymptomatic samples. Nested PCR revealed that SCWL phytoplasma was detected in all
493 plant samples presenting white leaf symptoms and in 234 of 287 samples without white leaf symptoms; thus, the SCWL phytoplasma detection rate was $81.53 \%$ in asymptomatic samples. In terms of varieties, the SCWL phytoplasma detection rate of asymptomatic samples of Liucheng05-136 was 100\% (11/11), and that of asymptomatic samples of Yingyu91-59 was the lowest, at 70.93\% (61/ 86) (Fig. 2).

Sequence analysis of nested PCR products. The sequencing of 26 nested PCR products showed that all amplicons (GenBank accession nos. MK962442 to MK962467) were 1,247 bp long and shared $100 \%$ nucleotide sequence identity. BLASTn analysis showed that all sequences shared $100 \%$ nucleotide sequence identity with the 16S rRNA gene sequences of SCWL phytoplasma (GenBank accession nos. KR020691 and KR020692) from Lincang, Yunnan province.

\section{Discussion}

In recent years, SCWL has been identified in many sugarcane planting areas in Yunnan province, China, and has become an extremely serious problem in some sugarcane growing areas. The prevalence of SCWL has become a major obstacle to the sustainable, healthy, and efficient development of the Yunnan sugarcane industry. In this study, we surveyed the incidence of SCWL in the field in Mangweng and Hepai in Yunnan province, which were the areas most affected by SCWL. The symptomatic incidence of SCWL in seven major sugarcane varieties differed significantly, but the positive PCR detection rate exceeded $90 \%$.

Studies have shown that not all sugarcane infected by SCWL phytoplasma shows white leaf symptoms, and the severity of these symptoms is affected by seed cane quality, sugarcane growth, cultivation measures, sugarcane variety, soil types and fertility, as well as the content of pathogens in sugarcane (Wongkaew 2012). In winter or at relatively low temperatures, the white leaf symptoms are masked, whereas in warm seasons or when the temperature is relatively high, white leaf symptoms will manifest rapidly (Wongkaew 2012). In sugarcane seedlings or plants inoculated with insect vectors, white leaf symptoms disappear shortly after their initial appearance (Ling 1962). Some of the diseased plants whose leaves are whitened without excessive tillering can

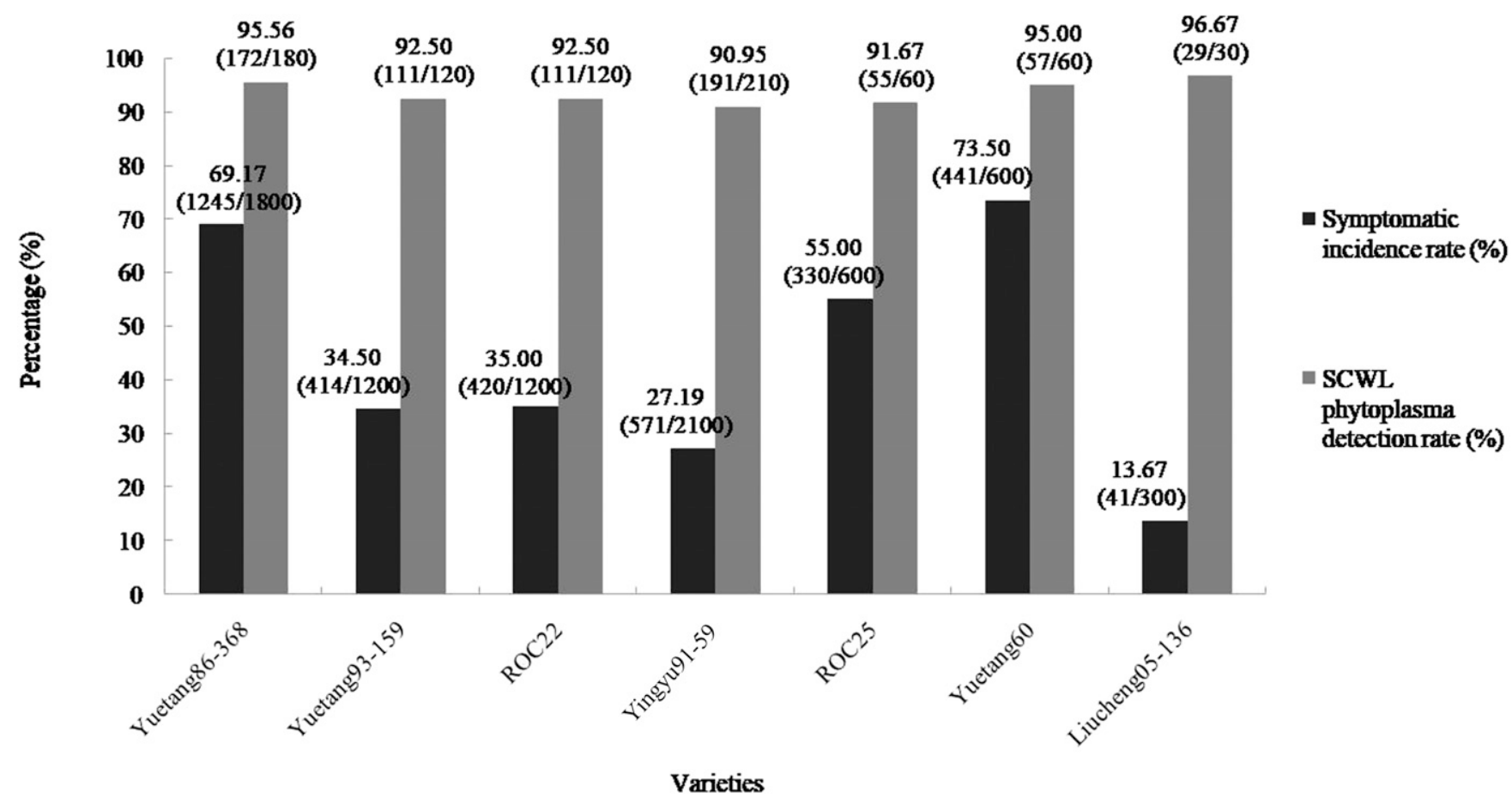

Fig. 1. Occurrence of sugarcane white leaf (SCWL) in major sugarcane varieties. 
even recover the appearance of healthy plants (Li et al. 2014a). Therefore, judging the incidence of SCWL infection by only observing the white leaf symptoms is an unreliable index of infection and does not accurately reflect the severity of SCWL infection. Therefore, the incidence of SCWL infection must be accurately detected molecularly. In this study, the mean SCWL incidence in the field was $44.38 \%$, but the mean SCWL phytoplasma detection rate using nested PCR was $93.08 \%$, and that of asymptomatic samples was $81.53 \%$. The symptomatic incidence of SCWL in some plots in the survey was only $7.3 \%$, but the SCWL phytoplasma detection rate was as high as $90 \%$. These data demonstrate that asymptomatic sugarcane might not necessarily be healthy.

Although the cultivation of disease-resistant varieties is the optimal measure to control the occurrence of diseases, no SCWLresistant varieties have been identified to date. Leu (1974) assessed the disease resistance of at least 400 sugarcane varieties, including wild sugarcane germplasm, using the insect transmission method, and found no resistant varieties. Similarly, no disease-resistant varieties were found among 158 sugarcane varieties tested in Thailand in 1988 to 1991 (Wongkaew 2012). In 2004 to 2007, the disease resistance of all sugarcane varieties, including their hybrid progenies and wild sugarcane germplasm, was evaluated, and again no SCWLresistant varieties were found (Wongkaew 2012). Subsequently, researchers evaluated disease resistance among different varieties in various sugarcane research institutions, but no resistant varieties to SCWL phytoplasma have been reported (Wongkaew 2012). In China, previous observations showed that the main varieties, including ROC10, ROC22, ROC25, Yuetang00-236, Yuetang93-159, Yuetang86-368, Yunzhe03-194, Yunzhe86-161, and Guitang12, were infected with SCWL (in particular, ROC22 and Yuetang86368) (Huang et al. 2018; Li et al. 2013). In this study, we surveyed seven local varieties and found that Yuetang60, Yuetang86-368, and ROC25 had a higher mean SCWL symptomatic incidence in the field, which exceeded $50 \%$. In contrast to previous studies (Li et al. 2013), the incidence of SCWL in ROC22 was lower than expected. Nested PCR showed that the SCWL phytoplasma detection rate of all sugarcane varieties was more than $90 \%$. These results showed that different symptom expressions are present between different sugarcane varieties. Because no uniform evaluation criteria exist for the disease tolerance of SCWL phytoplasma, it remains to be analyzed in more detail whether the characteristics of diseased plants that do not display symptoms can be used as an index for evaluating disease tolerance.

Because SCWL phytoplasma colonizes the phloem of sugarcane, the disease can easily spread via the propagation of sugarcane stalks. The major cause of SCWL outbreaks is the planting of diseased seed cane in sugarcane production. SCWL can also be transmitted by leafhoppers, and studies have shown that spraying with insecticides is not effective in reducing the incidence of SCWL (Leu 1974; Wangkeeree et al. 2012; Wongkaew 2012). Similarly, hot water treatment does not effectively eradicate SCWL phytoplasma from sugarcane stalks (Shan et al. 2013). At present, the production of healthy sugarcane seed cane by meristematic tissue culture is considered to be the most effective way to eliminate SCWL phytoplasma (Huang et al. 2018). The effective control of SCWL in Thailand has been achieved using diseasefree tissue-cultured seed canes (Wongkaew and Fletcher 2004), and it was also found that farmers ignored the potential danger of SCWL and its transmission mode when selecting seed canes, which was an important reason for the high incidence of SCWL in newly planted sugarcane. The introduction of disease-free seed cane and monitoring seed cane via PCR assays within the areas affected by SCWL are highly recommended. Likewise, quarantines must be instituted to prevent the spread of infected seed cane from these SCWL affected areas.

These results can form a basis for the evaluation and screening of resistant sugarcane varieties and the establishment of resistance grading standards, and also provide technical support for the rational distribution of sugarcane varieties and SCWL control.

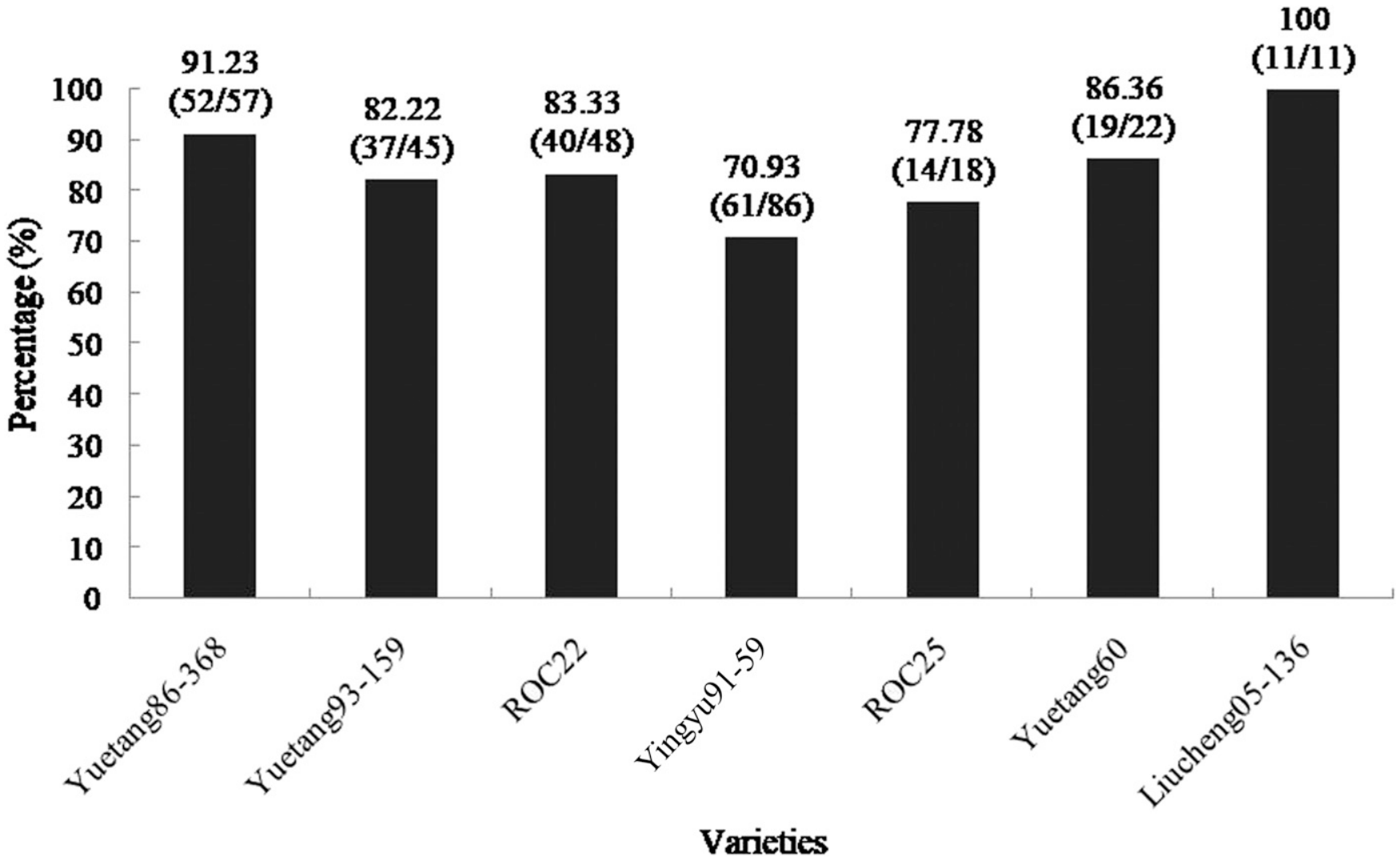

Fig. 2. Sugarcane white leaf phytoplasma detection rate of asymptomatic samples of different sugarcane varieties. 


\section{Literature Cited}

Hanboonsong, Y., Choosai, C., Panyim, S., and Damak, S. 2002. Transovarial transmission of sugarcane white leaf phytoplasma in the insect vector Matsumuratettix hiroglyphicus (Matsumura). Insect Mol. Biol. 11:97-103.

Hanboonsong, Y., Ritthison, W., Choosai, C., and Sirithorn, P. 2006. Transmission of sugarcane white leaf phytoplasma by Yamatotettix flavovittatus, a new leafhopper vector. J. Econ. Entomol. 99:1531-1537.

Huang, Y. K., Li, W. F., Zhang, R. Y., and Wang, X. Y. 2018. Color Illustration of Diagnosis and Control for Modern Sugarcane Diseases, Pests, and Weeds. Springer Nature Singapore, Singapore.

Kumarasinghe, N. C., and Jones, P. 2001. Identification of white leaf disease of sugarcane in Sri Lanka. Sugar Tech 3:55-58.

Lee, I., Gundersenrindal, D. E., Davis, R. E., and Bartoszyk, I. M. 1998. Revised classification scheme of phytoplasmas based on RFLP analyses of 16S rRNA and ribosomal protein gene sequences. Int. J. Syst. Bacteriol. 48:1153-1169.

Leu, L. S. 1974. An insectary method for testing sugarcane varieties for resistance to white leaf disease. Pages 266-274 in: Proceedings of the XV International Society of Sugarcane Technologists Congress. International Society of Sugarcane Technologists, Durban, South Africa.

Li, W. F., Shan, H. L., Huang, Y. K., Wang, X. Y., Zhang, R. Y., Luo, Z. M., Yin, J., and Shen, K. 2014a. Occurrence and control strategies of quarantine disease sugarcane white leaf. Sugar Crops China (3):66-68 (in Chinese with English abstract).

Li, W. F., Wang, X. Y., Huang, Y. K., Shan, H. L., Shen, K., Luo, Z. M., Zhang, R. Y., and Yin, J. 2014b. A quarantining sugarcane white leaf disease caused by phytoplasma found in sugarcane field in Yunnan. Acta Phytopathol. Sin. 44: 556-560 (in Chinese with English abstract).

Li, W. F., Wang, X. Y., Huang, Y. K., Shen, K., Shan, H. L., Luo, Z. M., Yin, J., Jia, Y. M., Bai, Z. G., and Zhang, R. Y. 2013. First report of sugarcane white leaf phytoplasma in Yunnan province, China. Can. J. Plant Pathol. 35:407-410.

Ling, K. C. 1962. White leaf disease of sugarcane. Taiwan Sugar (9):1-5.

Marcone, C. 2002. Phytoplasma diseases of sugarcane. Sugar Tech 4:79-85.

Rao, G. P., Priya, M., Thorat, V., and Manimekalai, R. 2017. A century progress of research on phytoplasma diseases in India. Phytopathogenic Mollicutes 7:1-38.

Shan, H. L., Li, W. F., Huang, Y. K., Wang, X. Y., Zhang, R. Y., Luo, Z. M., Shen, K., and Yin, J. 2013. The effect of hot-water treatment on eliminating sugarcane white leaf phytoplasma. J. Yunnan Agric. Univ. 28:438-441 (in Chinese with English abstract).

Smart, C. D., Schneider, B., Blomquist, C. L., Guerra, L. J., Harrison, N. A., Ahrens, U., Lorenz, K. H., Seemüller, E., and Kirkpatrick, B. C. 1996. Phytoplasma-specific PCR primers based on sequences of the 16S-23S rRNA spacer region. Appl. Environ. Microbiol. 62:2988-2993.

Thein, M. M., Jamjanya, T., Kobori, Y., and Hanboonsong, Y. 2012. Dispersal of leafhoppers Matsumuratettix hiroglyphicus and Yamatotettix flavovittatus (Homoptera: Cicadellidae), vectors of sugarcane white leaf disease. Appl. Entomol. Zool. 47:255-262.

Tiwari, A. K., Kumar, S., Mall, S., Jadon, V., and Rao, G. P. 2017. New efficient natural leafhopper vectors of sugarcane grassy shoot phytoplasma in India. Sugar Tech 19:191-197.

Wang, X. Y., Li, W. F., Huang, Y. K., Zhang, R. Y., Shan, H. L., Luo, Z. M., Yin, J., and Shen, K. 2015. Identification of sugarcane white leaf phytoplasma in fields and quaratine sugarcane samples in Yunnan province, China. Sugar Tech 17:85-88

Wangkeeree, J., Miller, T. A., and Hanboonsong, Y. 2012. Candidates for symbiotic control of sugarcane white leaf disease. Appl. Environ. Microbiol. 78:6804-6811.

Wongkaew, P. 2012. Sugarcane white leaf disease characterization, diagnosis development, and control strategies. Funct. Plant Sci. Biotechnol. 6:73-84.

Wongkaew, P., and Fletcher, J. 2004. Sugarcane white leaf phytoplasma in tissue culture: Long-term maintenance, transmission, and oxytetracycline remission. Plant Cell Rep. 23:426-434.

Wongkaew, P., Hanboonsong, Y., Sirithorn, P., Choosai, C., Boonkrong, S., Tinnangwattana, T., Kitchareonpanya, R., and Damak, S. 1997. Differentiation of phytoplasmas associated with sugarcane and gramineous weed white leaf disease and sugarcane grassy shoot disease by RFLP and sequencing. Theor. Appl. Genet. 95:660-663.

Zhang, R. Y., Li, W. F., Huang, Y. K., Wang, X. Y., Shan, H. L., Li, J., Cang, X. Y., Luo, Z. M., and Yin, J. 2019. Molecular identification of sugarcane white leaf in Puer, Yunnan province, China. Sugar Tech 21:734-736.

Zhang, R. Y., Li, W. F., Huang, Y. K., Wang, X. Y., Shan, H. L., Luo, Z. M., and Yin, J. 2016. Group 16SrXI phytoplasma strains, including subgroup 16SrXI-B and a new subgroup, 16SrXI-D, are associated with sugarcane white leaf. Int. J. Syst. Evol. Microbiol. 66:487-491. 